\title{
Numerical Analysis and Optimization of Internal Flow Field in Optical Module of DUV Laser
}

\author{
Bin LIU ${ }^{1,2, a}$, Jin-Bin DING ${ }^{1}$, Kui-Bo WANG ${ }^{1}$, Peng-Fei SHA ${ }^{1}$, Yi ZHOU ${ }^{1}$ and Yu \\ WANG $^{1}$
}

${ }^{1}$ Academy of Opto-electronics, Chinese Academy of Science, 100094 Beijing, China

2University of Chinese Academy of Science, 100094 Beijing, China

aliubin@aoe.ac.cn

Keywords: CFD, DUV laser, Flow field.

\begin{abstract}
In this paper, the internal flow field in an optical module of DUV laser was analyzed by ANSYS Fluent based on the finite volume method. The way of purging nitrogen was optimized according to the results of the analysis. After the optimization, a better nitrogen purge method was obtained, and a flow field with uniform velocity and low turbulence energy was obtained on the laser transmission path. The results of this paper can provide a reference for the design of the gas purge system of DUV laser.
\end{abstract}

\section{Introduction}

There are some problems when DUV laser trasmittes in the air. First of all, oxygen molelules will be ionized to produced ozone which is harmful to humans when the concentration is high. Second, a large number of energy will loss and the laser beam will be disturbed by airflow, which will reduce the laser transmission efficiency and beam quality. Finally, the optical elements exposed to the air are susceptible to contamination. Therefore, the exteral optical components of DUV lasers are always sealed in variety of optical modules which usually filled by protective gas, mostly nitrogen[1]. Optical module purification technology was presented in many patents[2][3][4], and precise pressure control technology was studied[5]. However, the existing research was limited to the study of gas supply pressure control, and did not involve optical module internal flow study and optimization. In fact, improper purge mode or flow rate, wouldn't help to improve the DUV laser transmission efficiency and beam quality, or even cause bad effects. In this paper, CFD software was used to analyze the nitrogen injection process of an optical module, and more reasonable nitrogen purge mode was found.

\section{Object of Study}

The object of this paper is based on an optical module which is actually used in a DUV laser. This module is used to protect the output coupling mirror (OC mirror) of laser resonator cavity, has some versatility. The geometric model of the optical module are shown in Fig1.

The inlets and outlets are circular holes with a diameter of $5 \mathrm{~mm}$. The rate of gas inject was 1 $\mathrm{L} / \mathrm{min}$, ie the inlet flow rate was $0.85 \mathrm{~m} / \mathrm{s}$, regardless of temperature.

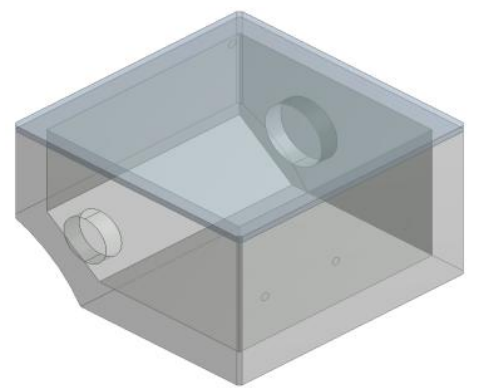

Figure 1. Geometric model and parameters 
The gas flow field is evaluated by the velocity uniformity and the turbulence kinetic energy, uniform gas flow and low turbulent kinetic energy are desirable.

\section{Computational Grid}

Firstly, the computational domain was divided into tetrahedral cells by using the ANSYS meshing tool. The tetrahedron grid is then transformed into polyhedral mesh by Fluent Solver. The polyhedral mesh has the characteristics of high precision and easy convergence[6]. The same computational precision as the tetrahedral grid could be obtained with $1 / 4$ number of polyhedral grids, $1 / 2$ of the memory, and 1/10 of the computation time. According to different structure, sevral parts of the local grid were rifined selectively, so the total quantity of cells was about 70000 . Figure 2 shows the polyhedral mesh of the computational domain.

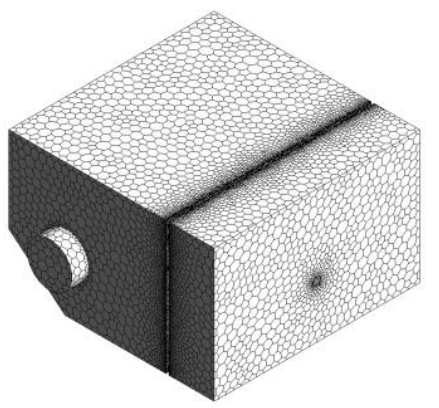

Figure 2. Computational grids

\section{Calculation Setting and Boundary Conditions}

ANSYS Fluent software was used to calculate the cases. Pressure based steady solver and standard k-epsilon turbulence model was choosen, and energy equation solver was activated. The pressurevelocity coupling scheme was SIMPLE, and the spatial discretization method were kept default state. The inlet hole was set to be a velocity inlet boundary, while the outlet hole was a pressure outlet.

\section{Calculation Results}

\section{Case1: Original Purge Way}

In order to make the nitrogen gas stream flows through the entire module, the inlet and out were provided at both diagonals of the optical module, as shown in Fig3.

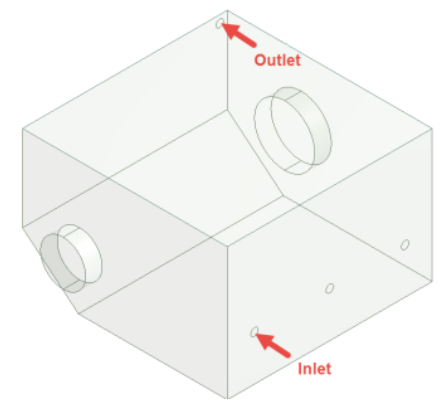

Figure 3. Inlet and outlet of case 1

The distribution of the nitrogen velocity in the horizontal section where the optical axis is located is shown in Fig4. 

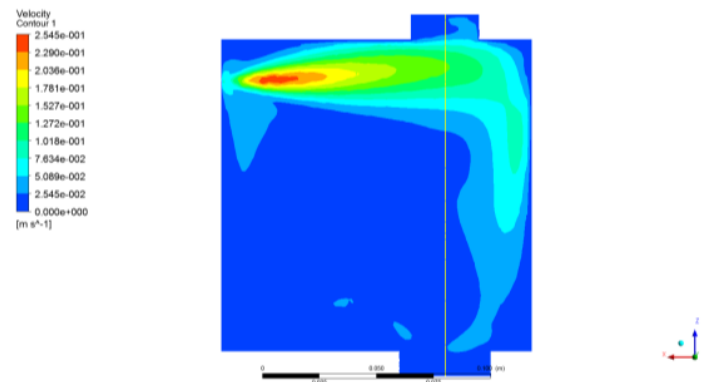

Figure 4. The distribution of the nitrogen velocity in optic axis horizontal section (Case 1)

The velocity distribution and the turbulent kinetic energy distribution on the optical axis of case1 are shown in Fig5. It can be seen that the velocity distribution on the optical path is very uneven under this purge mode. And the maximum velocity is $0.13 \mathrm{~m} / \mathrm{s}$, the laser beam may be disturbed. Experimental results also show that this kind of purge way didn't help to improve the energy and stability.
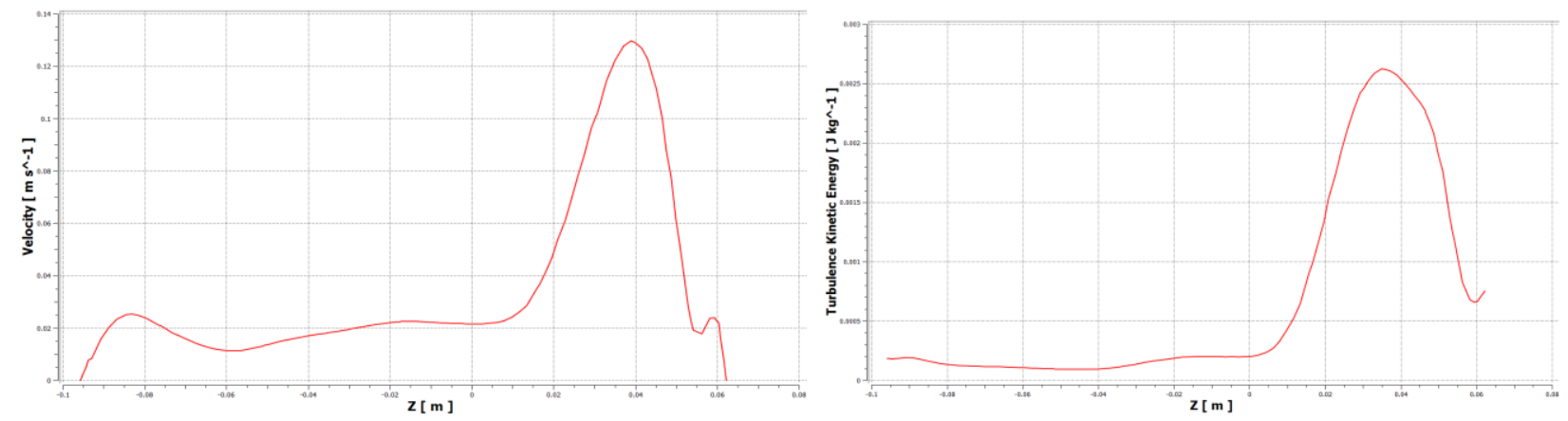

Figure 5. The velocity and turbulence kinetic energy distributions on the optical axis (Case 1)

\section{Case2: With a Gas Distribution Baffle Inside}

In order to improve the uniformity of the velocity and turbulence kinetic energy, a gas distribution baffle was designed to put inside the optical module, as shown in Fig6.

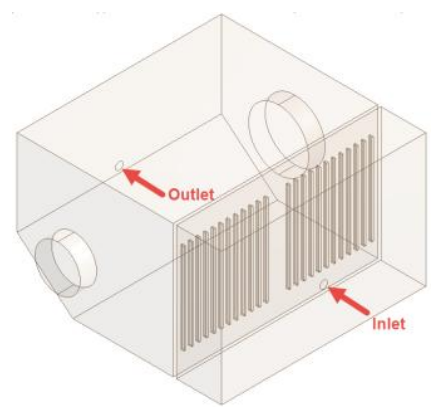

Figure 6. Inlet and outlet of case2

There are 24 rectangular holes on the baffle. The length and width of these holes are $60 \mathrm{~mm}$ and $1 \mathrm{~mm}$. The gas flow will be blocked by the baffle, then go through the holes on both sides.

According to the simulation results, the distribution of the nitrogen velocity in the horizontal section where the optical axis is located is shown in Fig7. 

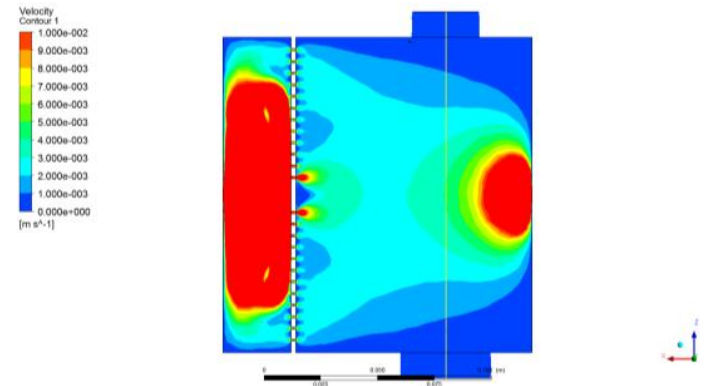

Figure 7. The distribution of the nitrogen velocity in optic axis horizontal section (Case 2)

It can be found that higer velocity gas was blocked by the baffle, and the gas was homogenized behind the baffle.

The velocity distribution and the turbulence kinetic energy distribution on the optical axis of case2 are shown in Fig8. The nitrogen flow velocity on the optical axis was reduced by an order of magnitude, while the turbulence kinetic energy reduced by two orders of magnitude. Because the gas was easier to pass through the middle holes, the gas velocity and turbulence kinetic energy in the middle were higher than both sides.
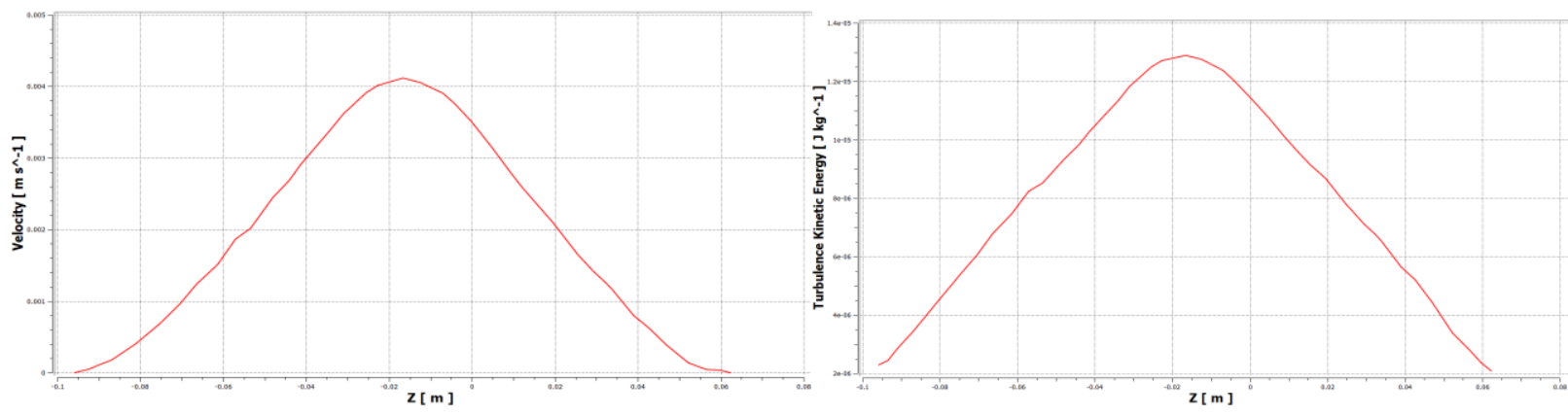

Figure 8 . The velocity and turbulence kinetic energy distributions on the optical axis (Case 2)

\section{Case3: With a Improved Gas Distribution Baffle Inside}

According the results above, the design of gas distribution baffle was improved, as shown in Fig9. Some rectangular holes in the middle were delete, and the length of the holes near the middle were reduced.

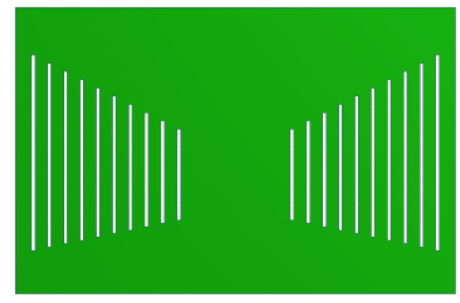

Figure 9. Improved gas distribution baffle

The simulation results were very similar to case 2 , while the uniformity of gas velocity and turbulence kinetic energy was much better, as shown in Fig10. 

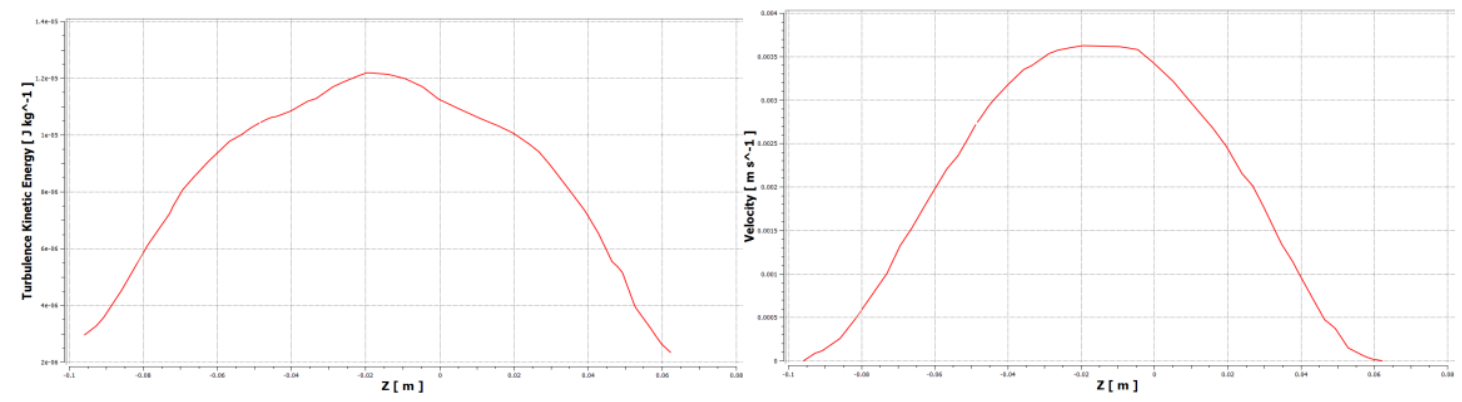

Figure 10. The velocity and turbulence kinetic energy distributions on the optical axis (Case 3)

\section{Case4: With More Outlets}

Increase the number of outlets, as shown in Fig11, so that less gas will flow through the milldle outlets, which means more uniform gas flow on the optical axis.

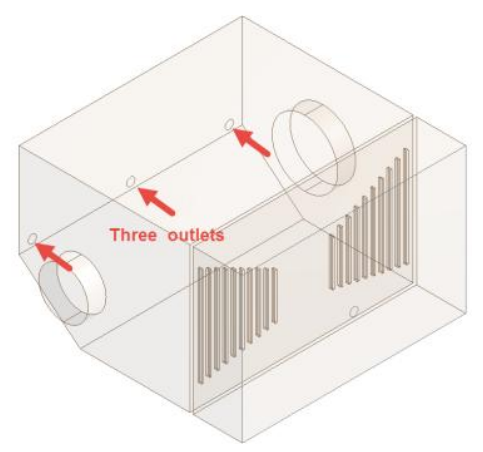

Figure 11. Three outlets model (Case 4)

Then we obtained the nitrogen velocity field as shown in Fig12, and The velocity distribution and turbulence kinetic energy distribution on the optical axis as shown in Fig13.
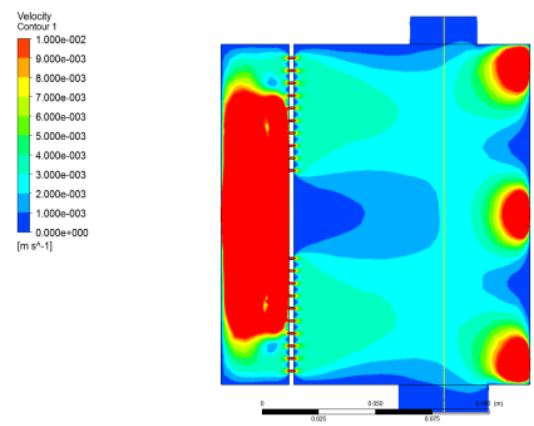

Figure 12. The distribution of the nitrogen velocity in optic axis horizontal section (Case 4)
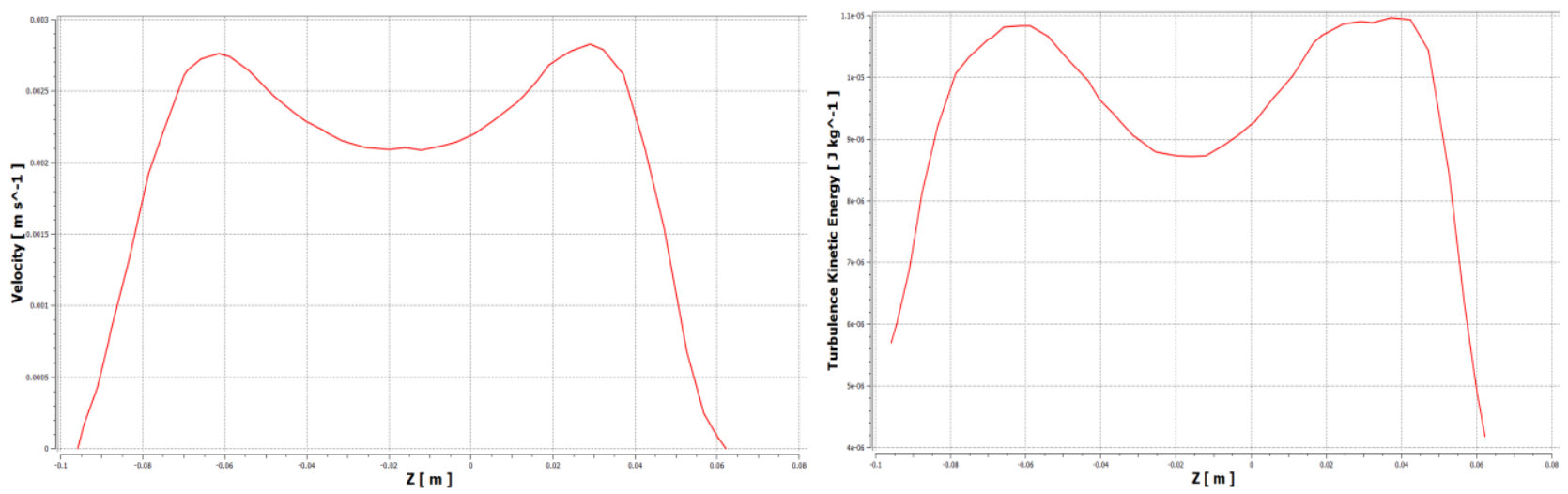

Figure 13. The velocity and turbulence kinetic energy distributions on the optical axis (Case 4) 
Therefore, a acceptable flow field was obtained as a result of using three outlets. The velocity and turbulence kinetic energy were kept on a very low level, which was good for purging DUV laser optical module.

\section{Conclusions}

In this paper, ANSYS Fluent software was used to analyze the flow field in an optical module, and the way of nitrogen purge was optimized step by step. According to the obtained results, the following conclusions can be drawn:1) A suitable gas distribution baffle can increase the uniformity of gas velociy and turbulence kinitic energy. 2) The magnitude of gas velocity and turbulence kinitic energy can be reduced a lot by distribution baffle. 3) The outlets design can affect the uniformity of gas velocity and turbulence kinitic energy on optical axis. These conclusions can also apply to the design of other gas purge module of DUV laser.

\section{References}

1. Haibo ZHANG, Qihong LOU, Jun ZHOU, et al. Arf excimer laser line narrow technique. Laser \& Optoelectronics Progress, 12, 46 51 ( 2009).

2. Rokni S, Tammadge D J, Watson T A. Fluorine control system for excimer lasers. U.S. Patent 5,978,406. (1999).

3. Azzola J H, Berger D, Duffey T P, et al. Gas discharge laser with gas temperature comtrol. U.S. Patent 6,034,978. (2000).

4. Binder M C, Carlesi J R, Das P P, et al. Automatic fluorine control system. U.S. Patent 6,151,349. (2000).

5. C. Melo, R.T.S. Ferreira, C. Boabaid Neto, et al. An experimental analysis of adiabatic capillary tubes. Applied Thermal Engineering, 19, (1999).

6. M. Peric. Flow simulation using control volumes of arbitrary polyhedral shape. ERCOFTAC Bulletin. 62, (2004). 